\title{
Dynamic Stress Intensity Factors for Three Parallel Cracks in an Infinite Plate Subject to Harmonic Stress Waves
}

\author{
Shouetsu Itou \\ Department of Mechanical Engineering, Kanagawa University, Yokohama, Japan \\ E-mail: itous001@kanagawa-u.ac.jp \\ Received February 25, 2010; revised April 9, 2010; accepted April 22, 2010
}

\begin{abstract}
Dynamic stresses around three parallel cracks in an infinite elastic plate that is subjected to incident time-harmonic stress waves normal to the cracks have been solved. Using the Fourier transform technique, the boundary conditions are reduced to six simultaneous integral equations. To solve these equations, the differences of displacements inside the cracks are expanded in a series. The unknown coefficients in those series are solved using the Schmidt method such that the conditions inside the cracks are satisfied. Numerical calculations are carried out for some crack configurations.
\end{abstract}

Keywords: Three Cracks, Time-Harmonic Problem, Stress Intensity Factor, Integral Equation, The Schmidt Method

\section{Introduction}

A time-harmonic solution for stresses around a crack in an infinite plate was reported by Loeber and Sih [1]. In their study, they obtained the Mode III dynamic stress intensity factor during the passage of a time-harmonic anti-plane shear wave. Subsequently, they also solved the crack problem for a compression wave and a vertically polarized shear wave [2]. Adopting a somewhat different approach, the same problem was studied independently by Mal [3]. The corresponding three-dimensional solutions for a penny-shaped crack have been obtained by Sih and Loeber [4,5] and by Mal [6].

Materials are generally weakened by some cracks. Therefore, it is of interest to reveal the mutual effect of the cracks on the dynamic stress intensity factors. Itou solved the dynamic stresses around two collinear cracks in which a self-equilibrated system of pressure is varied harmonically with time [7]. Later, the Mode III solution was given for two collinear cracks by Itou [8]. As for three collinear cracks, Mode I solutions were determined under the condition that time-harmonic normal traction is applied to the surfaces of the cracks [9].

Materials are occasionally weakened by some parallel cracks. Takakuda solved the time-harmonic problem for two parallel cracks in an infinite plane subjected to waves that impinge perpendicular to the cracks [10]. So and Huang analyzed the Mode III stress intensity factor around two cracks located in arbitrary positions in an infinite medium subjected to incident SH waves [11]. Meguid and Wang cleared the effect of the existence of an arbitrarily located and oriented micro defect on the dynamic stress intensity factors for a finite main crack subjected to a plane incident wave [12]. Ayatollahi and Fariborz provided the analysis of multiple curved cracks in an infinite plane under in-plane time harmonic loads [13]. Itou and Haliding assumed that two small collinear cracks are situated symmetrically above a main crack in an infinite plate and provided the dynamic stress intensity factors during passage of time-harmonic waves [14].

A peak value of the dynamic stress intensity factor $K_{i}^{\text {peak }}$ for collinear cracks in an infinite elastic plate is generally about 1.20-1.60 times larger than that of the corresponding static value $K_{i}^{\text {static }}$. However, in the paper [10], it was found that a peak value of the dynamic stress intensity factor for two parallel cracks is significantly larger than those for the collinear cracks. For example, for an infinite plate containing two parallel cracks of length $2 a$ separated by a distance $h$, the $K_{i}^{\text {peak }} /$ $K_{i}^{\text {static }}$ ratio is 4.16 for $h / a=1.0$ [10]. It was also shown that similar results appear for two parallel cracks in an infinite orthotropic plate subjected to incident time-harmonic stress waves [15]. From this fact, it is expected that $K_{i}^{\text {peak }} / K_{i}^{\text {static }}$ ratio will be very large for three parallel cracks during passage of the time harmonic stress waves. 
In investigating the $K_{i}^{\text {peak }} / K_{i}^{\text {static }}$ ratio for three parallel cracks, the mixed boundary value conditions are reduced to six dual integral equations. It has been shown that the integral equations can be converted into six sets of an infinite series and that the unknown coefficients in the series can be solved using the Schmidt method [16]. The author has developed a Fortran program to obtain the unknowns in four dual infinite series [15,17]. However, it is very difficult to write a Fortran program that is capable of solving the unknowns in six sets of an infinite series, making it difficult to solve the time-harmonic problem for three parallel cracks. The present author decided to solve the time-harmonic dynamic crack problem for three parallel cracks in an infinite elastic plate because it is of importance to provide the dynamic stress intensity factors in fracture mechanics.

In this study, time-harmonic stresses are solved for three parallel cracks in an infinite elastic plate during the passage of time-harmonic stress waves propagating normal to the cracks. The boundary conditions were reduced to dual integral equations with use of the Fourier transform technique. In order to solve these equations, the differences between the crack surface displacements are expanded to a series of functions that are equal to zero outside the cracks. The Schmidt method is modified so as to solve for the unknown coefficients in six sets of an infinite series.

A Fortran program has been developed to calculate the stress intensity factors for several crack configurations numerically.

\section{Fundamental Equations}

Consider a crack in an infinite plate located along the $x$-axis from $-a$ to $a$ at $y=0$, with respect to the rectangular coordinates $(x, y)$; an upper crack from $-b$ to $b$ at $y=h_{1}$; a lower crack from $-c$ to $c$ at $y=-h_{2}$; and incident time-harmonic stress waves propagating normally to the cracks, as shown in Figure 1. For convenience, $0<y<h_{1}$ is referred to as layer 1); $-h_{2}<y<0$ is referred to as layer 2); $h_{1}<y$ is referred to as the upper half-plane 3); and $y<-h_{2}$ is referred to as the lower half-plane 4). Let $u^{*}$ and $v^{*}$ be defined as the $x$ and $y$ components of the displacement, respectively. If the displacement components $u^{*}$ and $v^{*}$ are expressed by two functions $\phi^{*}(x, y, t)$ and $\varphi^{*}(x, y, t)$ such that

$$
u^{*}=\partial \phi^{*} / x-\partial \varphi^{*} \partial / \partial y, \quad v^{*}=\partial \varphi^{*} / \partial x+\partial \phi^{*} / \partial y
$$

the equations of motion reduce to the following forms:

$$
\begin{aligned}
& \partial^{2} \phi^{*} / \partial x^{2}+\partial^{2} \phi^{*} / \partial y^{2}=1 / c_{L}^{2} \times \partial^{2} \phi^{*} / \partial t^{2}, \\
& \partial^{2} \varphi^{*} / \partial x^{2}+\partial^{2} \varphi^{*} / \partial y^{2}=1 / c_{T}^{2} \times \partial^{2} \varphi^{*} / \partial t^{2}
\end{aligned}
$$

where $t$ is time. The dilatational wave velocity $c_{L}$ and the shear wave velocity $c_{T}$ under plane stress conditions can be given as follows:

$$
c_{L}^{2}=2 \mu /[(1-v) \rho], \quad c_{L}^{2}=\mu / \rho
$$

where $\mu$ is the modulus of rigidity, $v$ is Poisson's ratio, and $\rho$ is the density of the material.

The stresses can be expressed by the equations

$$
\begin{aligned}
& \tau_{y y}^{*} /(2 \mu)=-\partial^{2} \phi^{*} / \partial x^{2}+\kappa^{2} /\left(2 c_{L}^{2}\right) \partial^{2} \phi^{*} / \partial t^{2}+\partial^{2} \varphi^{*} / \partial x \partial y \\
& \tau_{x x}^{*} /(2 \mu)=-\partial^{2} \phi^{*} / \partial y^{2}+\kappa^{2} /\left(2 c_{L}^{2}\right) \partial^{2} \phi^{*} / \partial t^{2}-\partial^{2} \varphi^{*} / \partial x \partial y(4) \\
& \tau_{x y}^{*} /(2 \mu)=\partial^{2} \phi^{*} / \partial x \partial y+\partial^{2} \varphi^{*} / \partial x^{2}-\kappa^{2} /\left(2 c_{L}^{2}\right) \partial^{2} \phi^{*} / \partial t^{2}
\end{aligned}
$$

with

$$
\kappa^{2}=\left(c_{L} / c_{T}\right)^{2}=2 /(1-v)
$$

The incident stress waves that propagate through the infinite plate parallel to the $y$-axis in the negative direction can be expressed as follows:

$$
\begin{aligned}
& \tau_{y y}^{(i n c)^{*}}=p \exp \left[i\left(\omega t+\frac{\omega}{c_{L}} y\right)\right]=p \exp \left[i \frac{\omega}{c_{L}}\left(y+c_{L} t\right)\right](6) \\
& \tau_{x y}^{(i n c)^{*}}=0
\end{aligned}
$$

where $p$ is a constant and $\omega$ is the circular frequency.

Substituting the following relations

$$
\begin{aligned}
& \phi^{*}(x, y, t)=\phi \exp (i \omega t), \\
& \varphi^{*}(x, y, t)=\varphi \exp (i \omega t)
\end{aligned}
$$

into Equation (2) results in

$$
\begin{aligned}
& \partial^{2} \phi / \partial x^{2}+\partial^{2} \phi / \partial y^{2}+\alpha^{2} \phi=0, \\
& \partial^{2} \varphi / \partial x^{2}+\partial^{2} \varphi / \partial y^{2}+\beta^{2} \varphi=0
\end{aligned}
$$

with

$$
\begin{aligned}
\beta & =\omega / c_{T}, \\
\alpha & =\omega / c_{L}=\left(c_{T} / c_{L}\right) \times \omega / c_{T} \\
& =\sqrt{1 /\left(c_{L} / c_{T}\right)^{2}} \times \omega / c_{T}=(1 / \kappa) \times \beta
\end{aligned}
$$

Hereafter, the time factor $\exp (i \omega t)$ is omitted from the equations for convenience. Hence, displacements and stresses are expressed, respectively, by the following:

$$
\begin{aligned}
& u=\partial \varphi / \partial x-\partial \phi / \partial y \\
& v=\partial \phi / \partial x+\partial \varphi / \partial y
\end{aligned}
$$




$$
\begin{aligned}
& \tau_{y y} /(2 \mu)=-\partial^{2} \phi / \partial x^{2}-\kappa^{2} \omega^{2} /\left(2 c_{L}^{2}\right) \phi+\partial^{2} \varphi / \partial x \partial y \\
& \tau_{x x} /(2 \mu)=-\partial^{2} \phi^{*} / \partial y^{2}-\kappa^{2} \omega^{2} /\left(2 c_{L}^{2}\right) \phi-\partial^{2} \varphi / \partial x \partial y \\
& \tau_{x y} /(2 \mu)=\partial^{2} \phi / \partial x \partial y+\partial^{2} \varphi / \partial x^{2}+\kappa^{2} \omega^{2} /\left(2 c_{L}^{2}\right) \phi
\end{aligned}
$$

The boundary conditions for this problem can be expressed as

$$
\begin{gathered}
\tau_{y y 1}=\tau_{y y 3}, \quad \tau_{x y 1}=\tau_{x y 3} \text { at } y=h_{1}, \quad|x| \leq \infty \\
\tau_{y y 1}=\tau_{y y 2}, \quad \tau_{x y 1}=\tau_{x y 2} \text { at } y=0, \quad|x| \leq \infty \\
\tau_{y y 2}=\tau_{y y 4}, \quad \tau_{x y 2}=\tau_{x y 4} \text { at } y=-h_{2}, \quad|x| \leq \infty \\
\tau_{y y 1}=-p \exp \left(i \omega h_{1} / c_{L}\right), \quad \tau_{x y 1}=0 \quad \text { at } y=h_{1}, \quad|x| \leq b \\
u_{3}-u_{1}=0, \quad v_{3}-v_{1}=0, \quad \text { at } y=h_{1}, \quad b \leq|x| \\
\tau_{y y 1}=-p, \quad \tau_{x y 1}=0 \text { at } y=0, \quad|x| \leq a \\
u_{1}-u_{2}=0, \quad v_{1}-v_{2}=0, \quad \text { at } y=0, \quad a \leq|x| \\
=-p \exp \left(-i \omega h_{2} / c_{L}\right), \quad \tau_{x y 2}=0 \quad \text { at } y=-h_{2}, \quad|x| \leq c \\
\tau_{y y 2}
\end{gathered}
$$

where the subscript $i(=1,2)$ indicates the layer $i$, the subscript 3 indicates the upper half-plane (3), and the subscript 4 indicates the lower half-plane (4).

\section{Analysis}

To obtain a solution, the following Fourier transforms are introduced:

$$
\begin{aligned}
& \bar{f}(\xi)=\int_{-\infty}^{\infty} f(x) \exp (i \xi x) d x, \\
& f(x)=1 /(2 \pi i) \times \int_{-\infty}^{\infty} \bar{f}(\xi) \exp (-i \xi x) d \xi
\end{aligned}
$$

Applying Equaiton (18) to Equation (8), we obtain:

$$
\begin{aligned}
& d^{2} \bar{\phi} / d y^{2}+\left(\alpha^{2}-\xi^{2}\right) \bar{\phi}=0, \\
& d^{2} \bar{\varphi} / d y^{2}+\left(\beta^{2}-\xi^{2}\right) \bar{\varphi}=0
\end{aligned}
$$

In the Fourier transform domain, the displacements and stresses are denoted, respectively, by the forms

$$
\begin{gathered}
\bar{u}=-i \xi \bar{\phi}-d \bar{\varphi} / d y, \quad \bar{v}=d \bar{\phi} / d y-i \xi \bar{\varphi} \\
\bar{\tau}_{y y} /(2 \mu)=\xi^{2} \bar{\phi}-1 / 2 \times \kappa^{2} \alpha^{2} \bar{\phi}-i \xi d \bar{\varphi} / d y \\
\bar{\tau}_{x x} /(2 \mu)=-d^{2} \bar{\phi} / d y^{2}-1 / 2 \times \kappa^{2} \alpha^{2} \bar{\phi}+i \xi d \bar{\varphi} / d y \\
\bar{\tau}_{x y} /(2 \mu)=-i \xi d \bar{\phi} / d y-\xi^{2} \bar{\varphi}+1 / 2 \times \kappa^{2} \alpha^{2} \bar{\varphi}
\end{gathered}
$$

For the layer $i(=1,2)$, the solutions of Equation (22) have the following forms:

$$
\begin{aligned}
& \bar{\phi}_{i}=A_{i} \sinh \left(\sqrt{\xi^{2}-\alpha^{2}} y\right)+B_{i} \cosh \left(\sqrt{\xi^{2}-\alpha^{2}} y\right) \\
& \bar{\varphi}_{i}=C_{i} \sinh \left(\sqrt{\xi^{2}-\beta^{2}} y\right)+D_{i} \cosh \left(\sqrt{\xi^{2}-\beta^{2}} y\right)
\end{aligned}
$$

where $A_{i}, B_{i}, \cdots, D_{i}$ are unknown coefficients. For the upper half-plane (3) and the lower half-plane (4), the solutions of Equation (22) have the following forms in terms of the unknown coefficients $C_{3}, D_{3}, C_{4}, D_{4}$ :

$$
\begin{aligned}
& \bar{\phi}_{3}=C_{3} \exp \left(-\sqrt{\xi^{2}-\alpha^{2}} y\right)=C_{3} \exp \left(-i \sqrt{\alpha^{2}-\xi^{2}} y\right) \\
& \bar{\varphi}_{3}=D_{3} \exp \left(-\sqrt{\xi^{2}-\beta^{2}} y\right)=D_{3} \exp \left(-i \sqrt{\beta^{2}-\xi^{2}} y\right) \\
& \bar{\phi}_{4}=C_{4} \exp \left(\sqrt{\xi^{2}-\alpha^{2}} y\right)=C_{4} \exp \left(i \sqrt{\alpha^{2}-\xi^{2}} y\right) \\
& \bar{\varphi}_{4}=D_{4} \exp \left(\sqrt{\xi^{2}-\beta^{2}} y\right)=D_{4} \exp \left(i \sqrt{\beta^{2}-\xi^{2}} y\right)
\end{aligned}
$$

The stresses and displacements can be expressed by twelve unknowns: $A_{1}, B_{1}, C_{1}, D_{1}, A_{2}, B_{2}, C_{2}, D_{2}, C_{3}, D_{3}$, $C_{4}$ and $D_{4}$. Using Equations (12), (13) and (14), which are valid for $|x| \leq \infty$, the twelve unknowns are reduced to six unknowns, yielding the following relations:

$$
\begin{aligned}
& i C_{2}=A_{1} f_{11}+B_{1} f_{12}+i C_{1} f_{13}+i D_{1} f_{14}+A_{2} f_{15}+B_{2} f_{16} \\
& i D_{2}=A_{1} f_{21}+B_{1} f_{22}+i C_{1} f_{23}+i D_{1} f_{24}+A_{2} f_{25}+B_{2} f_{26} \\
& C_{3}=A_{1} f_{31}+B_{1} f_{32}+i C_{1} f_{33}+i D_{1} f_{34}+A_{2} f_{35}+B_{2} f_{36} \\
& i D_{3}=A_{1} f_{41}+B_{1} f_{42}+i C_{1} f_{43}+i D_{1} f_{44}+A_{2} f_{45}+B_{2} f_{46} \\
& C_{4}=A_{1} f_{51}+B_{1} f_{52}+i C_{1} f_{53}+i D_{1} f_{54}+A_{2} f_{55}+B_{2} f_{56} \\
& i D_{4}=A_{1} f_{61}+B_{1} f_{62}+i C_{1} f_{63}+i D_{1} f_{64}+A_{2} f_{65}+B_{2} f_{66}
\end{aligned}
$$

where the expressions of the known functions $f_{i j}(i, j=1,2, \cdots, 6)$ have been omitted.

To satisfy the boundary conditions (16), (18) and (20), the differences of the displacements are expanded as follows:

$$
\begin{aligned}
\pi\left(u_{1 b}-u_{3 b}\right) & =\sum_{n=1}^{\infty} a_{n} \sin \left[2 n \times \sin ^{-1}(x / b)\right] \text { for }|x| \leq b \\
& =0 \text { for } b \leq|x|
\end{aligned}
$$

$$
\begin{aligned}
\pi\left(v_{1 b}-v_{3 b}\right) & =\sum_{n=1}^{\infty} b_{n} \cos \left[(2 n-1) \sin ^{-1}(x / b)\right] \text { for }|x| \leq b \\
& =0 \text { for } b \leq|x| \\
\pi\left(u_{1 a}-u_{2 a}\right) & =\sum_{n=1}^{\infty} c_{n} \sin \left[2 n \times \sin ^{-1}(x / a)\right] \text { for }|x| \leq a \\
& =0 \text { for } a \leq|x|
\end{aligned}
$$




$$
\begin{aligned}
\pi\left(v_{1 a}-v_{2 a}\right) & =\sum_{n=1}^{\infty} d_{n} \cos \left[(2 n-1) \sin ^{-1}(x / a)\right] \text { for }|x| \leq a \\
& =0 \text { for } a \leq|x|
\end{aligned}
$$

$$
\begin{aligned}
\pi\left(u_{2 c}-u_{4 c}\right) & =\sum_{n=1}^{\infty} e_{n} \sin \left[2 n \times \sin ^{-1}(x / c)\right] \text { for }|x| \leq c \\
& =0 \text { for } c \leq|x|
\end{aligned}
$$

$$
\begin{aligned}
\pi\left(v_{2 c}-v_{4 c}\right) & =\sum_{n=1}^{\infty} f_{n} \cos \left[(2 n-1) \sin ^{-1}(x / c)\right] \text { for }|x| \leq c \\
& =0 \text { for } c \leq|x|
\end{aligned}
$$

where $a_{n}, b_{n}, c_{n}, d_{n}, e_{n}$ and $f_{n}$ are unknowns, and the subscripts $a, b$ and $c$ indicate the values at $y=h_{1}$, $y=0$ and $y=-h_{2}$, respectively. The Fourier transforms of Equations (29)-(34) are expressed by

$$
\begin{aligned}
& (-i)\left(\bar{u}_{1 b}-\bar{u}_{3 b}\right)=\sum_{n=1}^{\infty} a_{n}(2 n) / \xi \times J_{2 n}(b \xi) \\
& \left(\bar{v}_{1 b}-\bar{v}_{3 b}\right)=\sum_{n=1}^{\infty} b_{n}(2 n-1) / \xi \times J_{2 n-1}(b \xi) \\
& (-i)\left(\bar{u}_{1 a}-\bar{u}_{2 a}\right)=\sum_{n=1}^{\infty} c_{n}(2 n) / \xi \times J_{2 n}(a \xi) \\
& \left(\bar{v}_{1 a}-\bar{v}_{2 a}\right)=\sum_{n=1}^{\infty} d_{n}(2 n-1) / \xi \times J_{2 n-1}(a \xi) \\
& (-i)\left(\bar{u}_{2 c}-\bar{u}_{4 c}\right)=\sum_{n=1}^{\infty} e_{n}(2 n) / \xi \times J_{2 n}(c \xi) \\
& \left(\bar{v}_{2 c}-\bar{v}_{4 c}\right)=\sum_{n=1}^{\infty} f_{n}(2 n-1) / \xi \times J_{2 n-1}(c \xi)
\end{aligned}
$$

where $J_{n}(\xi)$ is the Bessel function.

However, the variables on the left-hand sides of Equations (35), (36) and (37) can be expressed in terms of the unknowns $A_{1}, B_{1}, i C_{1}, i D_{1}, A_{2}$ and $B_{2}$ :

$$
\begin{gathered}
(-i)\left(\bar{u}_{1 b}-\bar{u}_{3 b}\right)=A_{1} h_{11}+B_{1} h_{12}+i C_{1} h_{13}+i D_{1} h_{14}+A_{2} h_{15} \\
+B_{2} h_{16} \\
\left(\bar{v}_{1 b}-\bar{v}_{3 b}\right)=A_{1} h_{21}+B_{1} h_{22}+i C_{1} h_{23}+i D_{1} h_{24}+A_{2} h_{25}+B_{2} h_{26} \\
(-i)\left(\bar{u}_{1 a}-\bar{u}_{2 a}\right)=A_{1} h_{31}+B_{1} h_{32}+i C_{1} h_{33}+i D_{1} h_{34}+A_{2} h_{35} \\
+B_{2} h_{36} \\
\left(\bar{v}_{1 a}-\bar{v}_{2 a}\right)=A_{1} h_{41}+B_{1} h_{42}+i C_{1} h_{43}+i D_{1} h_{44}+A_{2} h_{45}+B_{2} h_{46}
\end{gathered}
$$

$$
\begin{gathered}
(-i)\left(\bar{u}_{2 c}-\bar{u}_{4 c}\right)=A_{1} h_{51}+B_{1} h_{52}+i C_{1} h_{53}+i D_{1} h_{54}+A_{2} h_{55} \\
+B_{2} h_{56} \\
\left(\bar{v}_{2 c}-\bar{v}_{4 c}\right)=A_{1} h_{61}+B_{1} h_{62}+i C_{1} h_{63}+i D_{1} h_{64}+A_{2} h_{65}+B_{2} h_{66}
\end{gathered}
$$

where the expressions of the known functions $h_{i j}(i, j=$ $1,2, \cdots, 6)$ have been omitted.

Equating Equations (35), (36) and (37) with Equations. (38), (39) and (40), the unknowns $A_{1}, B_{1}, i C_{1}, i D_{1}, A_{2}$ and $B_{2}$ can be indicated by the unknowns $a_{n}, b_{n}, c_{n}$, $d_{n}, e_{n}$ and $f_{n}$. For example, the unknown $A_{1}$ is expressed as:

$$
\begin{aligned}
A_{1} & =\sum_{n=1}^{\infty} a_{n} \times 2 n /(\xi \Delta) \times H_{11} J_{2 n}(b \xi)+\sum_{n=1}^{\infty} b_{n} \\
& \times(2 n-1) /(\xi \Delta) \times H_{21} J_{2 n}(b \xi) \\
& +\sum_{n=1}^{\infty} c_{n} \times 2 n /(\xi \Delta) \times H_{31} J_{2 n}(a \xi)+\sum_{n=1}^{\infty} d_{n} \\
& \times(2 n-1) /(\xi \Delta) \times H_{41} J_{2 n}(a \xi) \\
& +\sum_{n=1}^{\infty} e_{n} \times 2 n /(\xi \Delta) \times H_{51} J_{2 n}(c \xi) \\
& +\sum_{n=1}^{\infty} f_{n} \times(2 n-1) /(\xi \Delta) \times H_{61} J_{2 n}(a c \xi)
\end{aligned}
$$

where $H_{i j}$ is the cofactor of the element $h_{i j}$, and $\Delta$ is given by

$$
\Delta=\left|h_{i j}\right|, \quad(i, j=1,2, \cdots, 6)
$$

Consequently, stresses that satisfy the boundary conditions (12), (13), (14), (16), (18) and (20) can be expressed in terms of the unknowns $a_{n}, b_{n}, c_{n}, d_{n}, d_{n}$, and $f_{n}$. For example, stress $\tau_{y y 1}$ at $y=h_{1}$ is of the form

$$
\begin{aligned}
& \tau_{y y 1(h 1)}=\sum_{n=1}^{\infty} a_{n} \times(2 n) / \pi \times \int_{0}^{\infty} Q_{1}(\xi) J_{2 n}(\xi b) \cos (\xi x) d \xi \\
& \quad+\sum_{n=1}^{\infty} b_{n} \times(2 n-1) / \pi \times \int_{0}^{\infty} Q_{2}(\xi) J_{2 n-1}(\xi b) \cos (\xi x) d \xi \\
& \quad+\sum_{n=1}^{\infty} c_{n} \times(2 n) / \pi \times \int_{0}^{\infty} Q_{3}(\xi) J_{2 n}(\xi a) \cos (\xi x) d \xi \\
& \quad+\sum_{n=1}^{\infty} d_{n} \times(2 n-1) / \pi \times \int_{0}^{\infty} Q_{4}(\xi) J_{2 n-1}(\xi a) \cos (\xi x) d \xi \\
& \quad+\sum_{n=1}^{\infty} e_{n} \times(2 n) / \pi \times \int_{0}^{\infty} Q_{5}(\xi) J_{2 n}(\xi c) \cos (\xi x) d \xi \\
& \quad+\sum_{n=1}^{\infty} f_{n} \times(2 n-1) / \pi \times \int_{0}^{\infty} Q_{6}(\xi) J_{2 n-1}(\xi c) \cos (\xi x) d \xi
\end{aligned}
$$

where the expressions of the known functions $Q_{i}(\xi)$ 
$(i=1,2, \cdots, 6)$ have been omitted. Finally, the remaining boundary conditions (15), (17) and (19), which must be satisfied inside the cracks, reduce to the forms:

$$
\begin{gathered}
\sum_{n=1}^{\infty} a_{n} F_{n 11}(x)+\sum_{n=1}^{\infty} b_{n} F_{n 12}(x)+\sum_{n=1}^{\infty} c_{n} F_{n 13}(x)+\sum_{n=1}^{\infty} d_{n} F_{n 14}(x) \\
+\sum_{n=1}^{\infty} e_{n} F_{n 15}(x)+\sum_{n=1}^{\infty} f_{n} F_{n 16}(x)=-u_{1}(x) \text { for }|x| \leq b
\end{gathered}
$$$$
\sum_{n=1}^{\infty} a_{n} F_{n 21}(x)+\sum_{n=1}^{\infty} b_{n} F_{n 22}(x)+\sum_{n=1}^{\infty} c_{n} F_{n 23}(x)+\sum_{n=1}^{\infty} d_{n} F_{n 24}(x)
$$$$
+\sum_{n=1}^{\infty} e_{n} F_{n 25}(x)+\sum_{n=1}^{\infty} f_{n} F_{n 26}(x)=-u_{2}(x) \text { for }|x| \leq b
$$$$
\sum_{n=1}^{\infty} a_{n} F_{n 31}(x)+\sum_{n=1}^{\infty} b_{n} F_{n 32}(x)+\sum_{n=1}^{\infty} c_{n} F_{n 33}(x)+\sum_{n=1}^{\infty} d_{n} F_{n 34}(x)
$$$$
+\sum_{n=1}^{\infty} e_{n} F_{n 35}(x)+\sum_{n=1}^{\infty} f_{n} F_{n 36}(x)=-u_{3}(x) \text { for }|x| \leq a
$$$$
\sum_{n=1}^{\infty} a_{n} F_{n 41}(x)+\sum_{n=1}^{\infty} b_{n} F_{n 42}(x)+\sum_{n=1}^{\infty} c_{n} F_{n 43}(x)+\sum_{n=1}^{\infty} d_{n} F_{n 44}(x)
$$$$
+\sum_{n=1}^{\infty} e_{n} F_{n 45}(x)+\sum_{n=1}^{\infty} f_{n} F_{n 46}(x)=-u_{4}(x) \text { for }|x| \leq a
$$$$
\sum_{n=1}^{\infty} a_{n} F_{n 51}(x)+\sum_{n=1}^{\infty} b_{n} F_{n 52}(x)+\sum_{n=1}^{\infty} c_{n} F_{n 53}(x)+\sum_{n=1}^{\infty} d_{n} F_{n 54}(x)
$$$$
+\sum_{n=1}^{\infty} e_{n} F_{n 55}(x)+\sum_{n=1}^{\infty} f_{n} F_{n 56}(x)=-u_{5}(x) \text { for }|x| \leq c
$$

$$
\begin{gathered}
\sum_{n=1}^{\infty} a_{n} F_{n 61}(x)+\sum_{n=1}^{\infty} b_{n} F_{n 62}(x)+\sum_{n=1}^{\infty} c_{n} F_{n 63}(x)+\sum_{n=1}^{\infty} d_{n} F_{n 64}(x) \\
+\sum_{n=1}^{\infty} e_{n} F_{n 65}(x)+\sum_{n=1}^{\infty} f_{n} F_{n 66}(x)=-u_{6}(x) \text { for }|x| \leq c
\end{gathered}
$$

where the functions $F_{n i j}(x)(i, j=1,2, \cdots, 6)$ are known. For example, $F_{n 11}(x), F_{n 12}(x)$ and $F_{n 66}(x)$ are expressed as

$$
\begin{aligned}
& F_{n 11}(x)=2 n / \pi \times \int_{0}^{\infty} Q_{1}(\xi) J_{2 n}(b \xi) \cos (\xi x) d \xi \\
& F_{n 12}(x)=(2 n-1) / \pi \times\left\{\int_{0}^{\infty}\left[Q_{2}(\xi)-Q_{2 L}\right] J_{2 n-1}(b \xi)\right. \\
& \left.\cos (\xi x) d \xi+Q_{2 L} / \sqrt{b^{2}-x^{2}} \times \cos \left[(2 n-1) \sin ^{-1}(x / b)\right]\right\}
\end{aligned}
$$

$$
F_{n 66}(x)=(2 n-1) / \pi \times \int_{0}^{\infty} Q_{36}(\xi) J_{2 n-1}(c \xi) \sin (\xi x) d \xi
$$

where the constant $Q_{2 L}$ is given by

$$
Q_{2 L}=Q_{2}\left(\xi_{L}\right)
$$

where $\xi_{L}$ is a larger value of $\xi$. The functions $u_{i}(x)$ are denoted by the equations:

$$
\begin{aligned}
& u_{1}(x)=p \times \exp \left(i \alpha h_{1}\right), \quad u_{2}(x)=0, \quad u_{3}(x)=p, \\
& u_{4}(x)=0, \quad u_{5}(x)=p \times \exp \left(-i \alpha h_{2}\right), \quad u_{6}(x)=0
\end{aligned}
$$

The unknowns $a_{n}, b_{n}, c_{n}, d_{n}, e_{n}$ and $f_{n}$ in Equations (44), (45), (46), (47), (48) and (49) can now be solved using the Schmidt method described in Appendix A.

\section{Stress Intensity Factors}

Once the unknown coefficients $a_{n}, b_{n}, c_{n}, d_{n}, e_{n}$ and $f_{n}$ have been solved, all the stresses and displacements can likewise be solved. In fracture mechanics, it is important to determine the stress intensity factors defined from the stresses in the region near the crack ends. Using the relations

$$
\begin{aligned}
& \int_{0}^{\infty} J_{n}(a \xi)[\cos (\xi x), \sin (\xi x)] d \xi \\
& =\left\{-a^{n}\left(x^{2}-a^{2}\right)^{-1 / 2}\left[x+\left(x^{2}-a^{2}\right)^{1 / 2}\right]^{-n} \sin (n \pi),\right. \\
& \left.a^{n}\left(x^{2}-a^{2}\right)^{-1 / 2}\left[x+\left(x^{2}-a^{2}\right)^{1 / 2}\right]^{-n} \cos (n \pi)\right\} \text { for } a \leq x
\end{aligned}
$$

the stress intensity factors can be expressed as

$$
\begin{aligned}
K_{1}^{h 1} & =\lim _{x \rightarrow b+} \sqrt{2 \pi(x-b)} \tau_{y y 1(h 1)} \\
& =\sum_{n=1}^{\infty} b_{n} \times(2 n-1) \times(-1)^{n} Q_{2 L} / \sqrt{\pi b} \\
K_{2}^{h 1} & =\lim _{x \rightarrow b+} \sqrt{2 \pi(x-b)} \tau_{x y 1(h 1)} \\
& =\sum_{n=1}^{\infty} a_{n} \times 2 n \times(-1)^{n} Q_{7 L} / \sqrt{\pi b} \\
K_{1}^{0} & =\lim _{x \rightarrow a+} \sqrt{2 \pi(x-a)} \tau_{y y 1(0)} \\
& =\sum_{n=1}^{\infty} d_{n} \times(2 n-1) \times(-1)^{n} Q_{16 L} / \sqrt{\pi a} \\
K_{2}^{0} & =\lim _{x \rightarrow a+} \sqrt{2 \pi(x-a)} \tau_{x y 1(0)} \\
& =\sum_{n=1}^{\infty} c_{n} \times 2 n \times(-1)^{n} Q_{21 L} / \sqrt{\pi a}
\end{aligned}
$$




$$
\begin{aligned}
K_{1}^{-h 2} & =\lim _{x \rightarrow c+} \sqrt{2 \pi(x-c)} \tau_{y y 4(-h 2)} \\
& =\sum_{n=1}^{\infty} f_{n} \times(2 n-1) \times(-1)^{n} Q_{30 L} / \sqrt{\pi c} \\
K_{2}^{-h 2} & =\lim _{x \rightarrow c+} \sqrt{2 \pi(x-c)} \tau_{x y 4(-h 2)} \\
& =\sum_{n=1}^{\infty} e_{n} \times 2 n \times(-1)^{n} Q_{35 L} / \sqrt{\pi c}
\end{aligned}
$$

where the constants $Q_{i L}(i=7,16,21,30,35)$ are given by expressions taking the similar form as Equation (53).

\section{Numerical Examples}

The dynamic stress intensity factors were calculated numerically with quadruplex precision using a Fortran program, during the operation of which, overflow and underflow do not occur within the range $10^{-5500}$ to $10^{+5500}$. Numerical calculations were performed for a Poisson's ratio $v=0.25$.

The semi-infinite integrals, which appear in the known functions $F_{n i j}(x) \quad(i, j=1,2, \cdots, 6)$ must be evaluated numerically. It can be verified that the numerical integrations have been performed satisfactorily because the integrands decay rapidly as the integration variable $\xi$ increases. To solve the unknown coefficients $a_{n}, b_{n}$, $c_{n}, d_{n}, e_{n}$ and $f_{n}$, the Schmidt method has been applied by truncating the infinite series in Equations (44), (45), (46), (47), (48) and (49) by summing from $n=1$ to $n=8$. It has been verified that the values for the left-hand side of the equations coincide with those for the right-hand side with acceptable accuracy.

The absolute values of the stress intensity factors given in Equations (56), (57) and (58) are calculated for $b / a=c / a=1.0$ and $h_{1} / a=h_{2} / a=2.5$; these are plotted with respect to $\omega a / c_{T}$ in Figure 2. The straight dashed lines on the left-hand side of Figure 2 indicate the corresponding static values given by Ishida [18]. Figure 3 shows the values for $h_{1} / a=h_{2} / a=1.0$. The dynamic stress intensity factors for two cracks in an infinite plate have been solved by Takakuda [10]. In the present study, the same problem has been reworked, and the results are plotted in Figures $\mathbf{4}$ and $\mathbf{5}$ for $h_{1} / a=2.5$ and 1.0 , respectively, where $b / a=1.0$ is assumed, and $h_{1}$ denotes the distance between the two parallel cracks. For the case of two parallel cracks, the distance $h_{2}$ in Figure 1 is considered to be infinite.

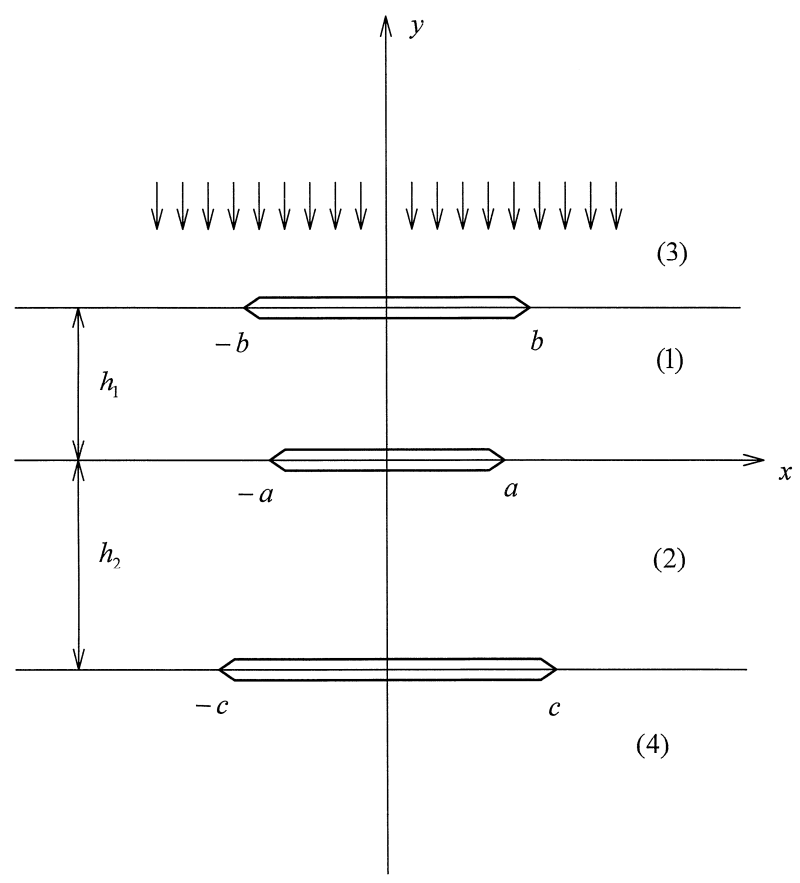

Figure 1. Geometry and coordinate system.

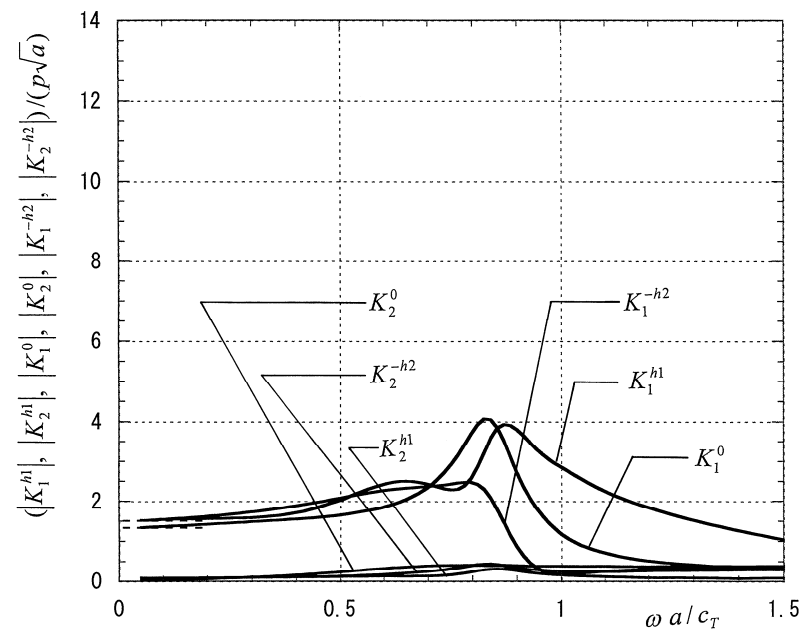

Figure 2. Stress intensity factors $\left|K_{1}^{h 1}\right|,\left|K_{2}^{h 1}\right|,\left|K_{1}^{0}\right|,\left|K_{2}^{0}\right|$, $\left|K_{1}^{-h 2}\right|$ and $\left|K_{2}^{-h 2}\right|$ for $h_{1} / a=h_{2} / a=2.5$ and $b / a=c / a=$ 1.0 .

\section{Discussion}

In the previous paper [14], time-harmonic stresses are solved for three cracks in an infinite elastic plate during the passage of time-harmonic stress waves. Two collinear cracks are situated symmetrically on either side of the main crack. The mixed boundary conditions with respect to the three cracks are reduced to four sets of an infinite series. The two sets of an infinite series are derived from 


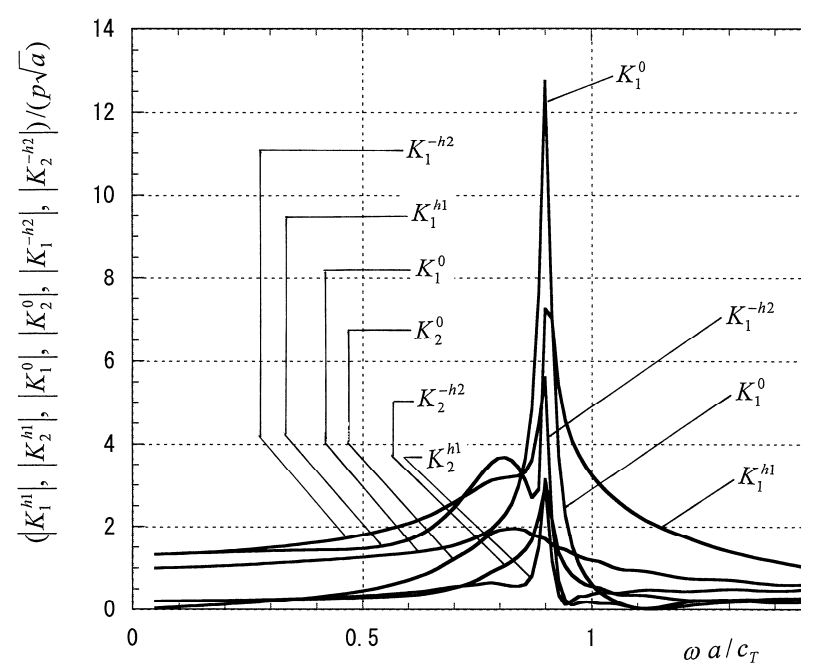

Figure 3. Stress intensity factors $\left|K_{1}^{h 1}\right|,\left|K_{2}^{h 1}\right|,\left|K_{1}^{0}\right|,\left|K_{2}^{0}\right|$, $\left|K_{1}^{-h 2}\right|$ and $\left|K_{2}^{-h 2}\right|$ for $h_{1} / a=h_{2} / a=1.0$ and $b / a=c / a=$ 1.0 .

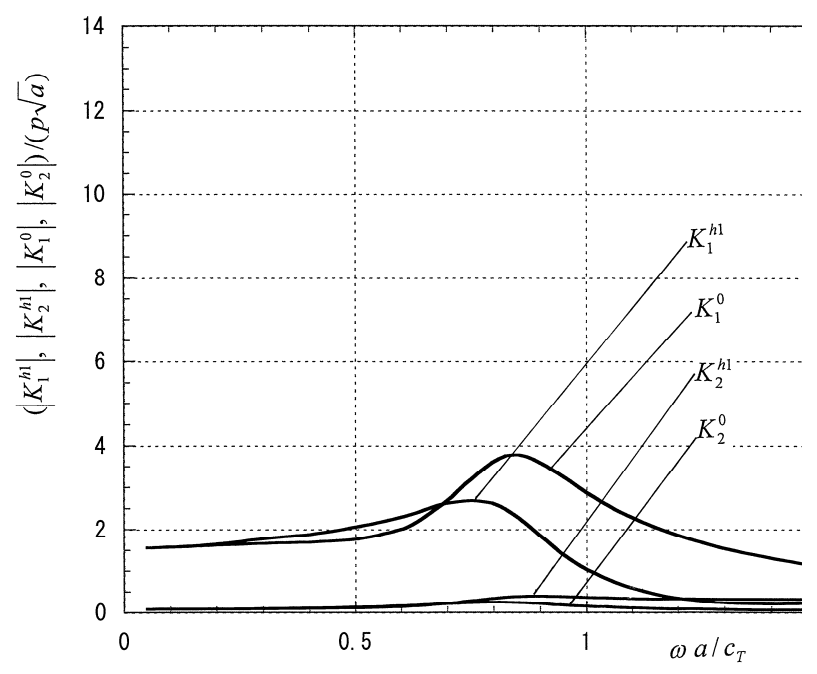

Figure 4. Stress intensity factors $\left|K_{1}^{h 1}\right|,\left|K_{2}^{h 1}\right|,\left|K_{1}^{0}\right|$ and $\left|K_{2}^{0}\right|$ for $h_{1} / a=2.5$ and $b / a=1.0$. (For the case of two parallel cracks, the distance $h_{2}$ in Figure 1 is considered to be infinite).

the boundary conditions inside the main crack, while the other two sets are derived from those inside one of the upper small cracks. The method to solve the unknown coefficients in the infinite series has been already described in $[15,17]$.

In the present paper, time-harmonic stresses are solved for an infinite elastic plate weakened by three parallel cracks. As the three cracks are not collinear, the boundary conditions with respect to the three cracks are reduced to six sets of an infinite series. Each of the two

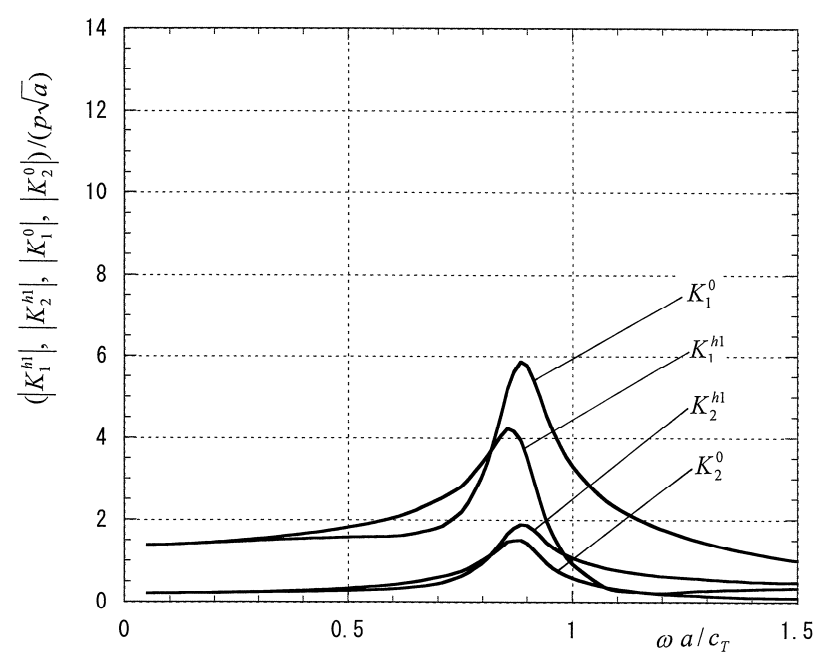

Figure 5. Stress intensity factors $\left|K_{1}^{h 1}\right|,\left|K_{2}^{h 1}\right|,\left|K_{1}^{0}\right|$ and $\left|K_{2}^{0}\right|$ for $h_{1} / a=1.0$ and $b / a=1.0$. (For the case of two parallel cracks, the distance $h_{2}$ in Figure 1 is considered to be infinite).

sets of an infinite series must satisfy the boundary conditions inside the lower, middle and upper cracks, respectively. Therefore, the Schmidt method is newly extended in the present paper to determine the six sets of unknown coefficients as described in section 1 .

In this study, the minimum value of $h_{1} / a\left(=h_{2} / a\right)$ used during numerical calculations was 1.0. If the calculations were performed for lower values of $h_{1} / a$ $\left(=h_{2} / a\right)$, the resulting peak value of $\left|K_{1}^{0} /(p \sqrt{a})\right|$ would be significantly larger than 12.8. There is scope for further inquiry into whether or not the absolute values of the stress intensity factors would increase in the case of four parallel cracks in an infinite elastic plate. The author considers that the peak value of $\left|K_{1}^{0} /(p \sqrt{a})\right|$ increases as the number of parallel cracks increases. If a timeharmonic load is applied to materials weakened by many parallel cracks, we note that the dynamic stress intensity factors would have a greater value than that for the corresponding static solution.

\section{Conclusions}

Based on the numerical calculations outlined above, and with reference to Figures 2 through 5, the following conclusions are reached:

1) There is a critical circular frequency in the three cracks case near $\omega a / c_{T}=0.9$ similar to that shown by Takakuda in the two-cracks case [10]. The value 
$\left|K_{1}^{0} /(p \sqrt{a})\right|$ is 12.8 for $h_{1} / a=h_{2} / a=1.0$. For two parallel cracks, the corresponding value is 5.87 . It can be seen that the presence of the third crack has a significant effect upon the dynamic stress intensity factor around the center crack between the upper and lower parallel cracks.

2) For $h_{1} / a=h_{2} / a=2.5$ (Figure 2), the slope to the peak value of $\left|K_{1}^{0} /(p \sqrt{a})\right|$ is comparatively gentle. However, for $h_{1} / a=h_{2} / a=1.0$ (Figure 3), the curve rises steeply to the maximum value of $\left|K_{1}^{0} /(p \sqrt{a})\right|$, after which it declines equally steeply. The curve exhibits a sharp peak at the critical circular frequency near $\omega a / c_{T}=0.9$.

3) In static solutions, the stress intensity factors for three equal-length cracks decrease slightly as $h_{1} / a$ $\left(=h_{2} / a\right)$ decreases. However, this is accompanied by a significant increase in the peak value of $\left|K_{1}^{0} /(p \sqrt{a})\right|$.

\section{References}

[1] J. F. Loeber and G. C. Sih, "Diffraction of Antiplane Shear by a Finite Crack," Journal of the Acoustical Society of America, Vol. 44, No. 1, 1968, pp. 90-98.

[2] G. C. Sih and J. F. Loeber, "Wave Propagation in an Elastic Solid with a Line of Discontinuity or Finite Crack," Quarterly of Applied Mathematics, Vol. 27, No. 2, 1969, pp. 193-213.

[3] A. K. Mal, "Interaction of Elastic Waves with a Griffith Crack," International Journal of Engineering Science, Vol. 8, No. 9, 1970, pp. 763-776.

[4] G. C. Sih and J. F. Loeber, "Torsional Vibration of an Elastic Solid Containing a Penny-Shaped Crack,” Journal of the Acoustical Society of America, Vol. 44, No. 5, 1968, pp. 1237-1245.

[5] G. C. Sih and J. F. Loeber, "Normal Compression and Radial Shear Waves Scattering at a Penny-Shaped Crack in an Elastic Solid," Journal of the Acoustical Society of America, Vol. 46, No. 3B, 1969, pp. 711-721.

[6] A. K. Mal, "Interaction of Elastic Waves with a PennyShaped Crack," International Journal of Engineering Science, Vol. 8, No. 5, 1970, pp. 381-388.
[7] S. Itou, "Dynamic Stress Concentration around Two Coplanar Griffith Cracks in an Infinite Elastic Medium," ASME Journal of Applied Mechanics, Vol. 45, No. 12, 1978, pp. 803-806.

[8] S. Itou, "Diffraction of an Antiplane Shear Wave by Two Coplanar Griffith Cracks in an Infinite Elastic Medium," International Journal of Solids and Structures, Vol. 16, No. 12, 1980, pp. 1147- 1153.

[9] S. Itou, "Dynamic Stresses around Two Cracks Placed Symmetrically to a Large Crack,” International Journal of Fracture, Vol. 75, No. 3, 1996, pp. 261-271.

[10] K. Takakuda, "Scattering of Plane Harmonic Waves by Cracks (in Japanese)," Transactions of Japan Society of Mechanical Engineeris, Series A, Vol. 48, No. 432, 1982, pp. 1014-1020.

[11] H. So and J. Y. Huang, "Determination of Dynamic Stress Intensity Factors of Two Finite Cracks at Arbitrary Positions by Dislocation Model,” International Journal of Engineering Science, Vol. 26, No. 2, 1988, pp. 111-119.

[12] S. A. Meguid and X. D. Wang, "On the Dynamic Interaction between a Microdefect and a Main Crack," Proceedings of the Royal Society of London, Series A, Vol. 448, No. 1934, 1995, pp. 449-464.

[13] M. Ayatollahi and S. J. Fariborz, "Elastodynamic Analysis of a Plane Weakened by Several Cracks," International Journal of Solids and Structures, Vol. 46, No. 7-8, 2009, pp. 1743-1754.

[14] S. Itou and H. Haliding, "Dynamic Stress Intensity Factors around Three Cracks in an Infinite Elastic Plane Subjected to Time-Hharmonic Stress Waves," International Journal of Fracture, Vol. 83, No. 4, 1997, pp. 379391.

[15] S. Itou and H. Haliding, "Dynamic Stress Intensity Factors around Two Parallel Cracks in an Infinite-Orthotropic Plane Subjected to Incident Harmonic Stress Waves," International Journal of Solids and Structures, Vol. 34, No. 9, 1997, pp. 1145-1165.

[16] P. M. Morse and H. Feshbach, "Methods of Theoretical Physics,” McGraw-Hill, New York, Vol. 1, 1958.

[17] S. Itou, “Axisymmetric Slipless Indentation of an Infinite Elastic Hollow Cylinder,” Bulletin of the Calcutta Mathematical Society, Vol. 68, No. 17, 1976, pp. 157-165.

[18] M. Ishida, "Elastic Analysis of Cracks and Stress Intensity Factors (in Japanese)," Fracture Mechanics and Strength of Materials, Baifuukan Press, Tokyo, Vol. 2, 1976. 


\section{Appendix A}

For convenience, Equations (42) through (47) can be rewritten as

$\sum_{n=1}^{\infty} a_{n} F_{n 11}(x)+\sum_{n=1}^{\infty} b_{n} F_{n 12}(x)+\sum_{n=1}^{\infty} c_{n} F_{n 13}(x)+\sum_{n=1}^{\infty} d_{n} F_{n 14}(x)$ $+\sum_{n=1}^{\infty} e_{n} F_{n 15}(x)+\sum_{n=1}^{\infty} f_{n} F_{n 16}(x)=-u_{1}(x)$ for $|x| \leq b$

$\sum_{n=1}^{\infty} a_{n} F_{n 21}(x)+\sum_{n=1}^{\infty} b_{n} F_{n 22}(x)+\sum_{n=1}^{\infty} c_{n} F_{n 23}(x)+\sum_{n=1}^{\infty} d_{n} F_{n 24}(x)$ $+\sum_{n=1}^{\infty} e_{n} F_{n 25}(x)+\sum_{n=1}^{\infty} f_{n} F_{n 26}(x)=-u_{2}(x)$ for $|x| \leq b$

$\sum_{n=1}^{\infty} a_{n} F_{n 31}(x)+\sum_{n=1}^{\infty} b_{n} F_{n 32}(x)+\sum_{n=1}^{\infty} c_{n} F_{n 33}(x)+\sum_{n=1}^{\infty} d_{n} F_{n 34}(x)$ $+\sum_{n=1}^{\infty} e_{n} F_{n 35}(x)+\sum_{n=1}^{\infty} f_{n} F_{n 36}(x)=-u_{3}(x)$ for $|x| \leq a$

$\sum_{n=1}^{\infty} a_{n} F_{n 41}(x)+\sum_{n=1}^{\infty} b_{n} F_{n 42}(x)+\sum_{n=1}^{\infty} c_{n} F_{n 43}(x)+\sum_{n=1}^{\infty} d_{n} F_{n 44}(x)$ $+\sum_{n=1}^{\infty} e_{n} F_{n 45}(x)+\sum_{n=1}^{\infty} f_{n} F_{n 46}(x)=-u_{4}(x)$ for $|x| \leq a$

$\sum_{n=1}^{\infty} a_{n} F_{n 51}(x)+\sum_{n=1}^{\infty} b_{n} F_{n 52}(x)+\sum_{n=1}^{\infty} c_{n} F_{n 53}(x)+\sum_{n=1}^{\infty} d_{n} F_{n 54}(x)$ $+\sum_{n=1}^{\infty} e_{n} F_{n 55}(x)+\sum_{n=1}^{\infty} f_{n} F_{n 56}(x)=-u_{5}(x)$ for $|x| \leq c$

$\sum_{n=1}^{\infty} a_{n} F_{n 61}(x)+\sum_{n=1}^{\infty} b_{n} F_{n 62}(x)+\sum_{n=1}^{\infty} c_{n} F_{n 63}(x)+\sum_{n=1}^{\infty} d_{n} F_{n 64}(x)$ $+\sum_{n=1}^{\infty} e_{n} F_{n 65}(x)+\sum_{n=1}^{\infty} f_{n} F_{n 66}(x)=-u_{6}(x)$ for $|x| \leq c$

A set of functions $G_{n}(x)$ that satisfy the orthogonality condition

$$
\int_{0}^{b} G_{m}(x) G_{n}(x) d x=I_{n} \times \delta_{m n}, \quad I_{n}=\int_{0}^{b} G_{n}^{2}(x) d x
$$

can be constructed from a given set of arbitrary function, say $F_{n 65}(x)$, such that

$$
G_{n}(x)=\sum_{i=1}^{n}\left(P_{i n} / P_{n n}\right) \times F_{i 65}(x)
$$

where $P_{i n}$ is the cofactor of the element $d_{i n}$ of $D_{n}{ }^{\prime}$, which is defined as

$$
D_{n}{ }^{\prime}=\left|\begin{array}{cccc}
d_{11} & d_{12} & \ldots & d_{1 n} \\
d_{21} & & \vdots \\
\vdots & & \vdots \\
d_{n 1} & \cdots \ldots \ldots & d_{n n}
\end{array}\right|, \quad d_{i n}=\int_{0}^{c} F_{i 65}(x) F_{n 65}(x) d x
$$

Representing the fifth series in Equation (A.6) by the orthogonal series $G_{n}(x)$ with coefficients $\varepsilon_{n}$, the following relationships are derivable:

$$
\begin{aligned}
& \sum_{n=1}^{\infty} e_{n} F_{n 65}(x)=\sum_{n=1}^{\infty} \varepsilon_{n} G_{n}(x) \\
& =-u_{6}(x)-\sum_{n=1}^{\infty} a_{n} F_{n 61}(x)-\sum_{n=1}^{\infty} b_{n} F_{n 62}(x)-\sum_{n=1}^{\infty} c_{n} F_{n 63}(x) \\
& -\sum_{n=1}^{\infty} d_{n} F_{n 64}(x)-\sum_{n=1}^{\infty} f_{n} F_{n 66}(x)
\end{aligned}
$$

The second equality yields

$$
\begin{aligned}
\varepsilon_{n} & =-\frac{1}{I_{n}}\left[\int_{0}^{c} G_{n}(x) u_{6}(x) d x+\sum_{i=1}^{\infty} a_{i} \int_{0}^{c} G_{n}(x) F_{i 61}(x) d x\right. \\
& +\sum_{i=1}^{\infty} b_{i} \int_{0}^{c} G_{n}(x) F_{i 62}(x) d x+\sum_{i=1}^{\infty} c_{i} \int_{0}^{c} G_{n}(x) F_{i 63}(x) d x \\
& \left.+\sum_{i=1}^{\infty} d_{i} \int_{0}^{c} G_{n}(x) F_{i 64}(x) d x+\sum_{i=1}^{\infty} f_{i} \int_{0}^{c} G_{n}(x) F_{i 66}(x) d x\right] \\
& \text { for }|x| \leq c
\end{aligned}
$$

and considering Equation (A.8), the first equality shows that

$e_{n}=\alpha_{n}^{0}+\sum_{i=1}^{\infty} a_{i} \alpha_{n i}^{a}+\sum_{i=1}^{\infty} b_{i} \alpha_{n i}^{b}+\sum_{i=1}^{\infty} c_{i} \alpha_{n i}^{c}+\sum_{i=1}^{\infty} d_{i} \alpha_{n i}^{d}+\sum_{i=1}^{\infty} f_{i} \alpha_{n i}^{f}$

with

$$
\begin{aligned}
& \alpha_{n}^{0}=-\sum_{j=n}^{\infty} \frac{P_{n j}}{P_{j j} I_{j}} \int_{0}^{c} G_{j}(x) u_{6}(x) d x, \\
& \alpha_{n i}^{a}=-\sum_{j=n}^{\infty} \frac{P_{n j}}{P_{j j} I_{j}} \int_{0}^{c} G_{j}(x) F_{i 61}(x) d x, \\
& \alpha_{n i}^{b}=-\sum_{j=n}^{\infty} \frac{P_{n j}}{P_{j j} I_{j}} \int_{0}^{c} G_{j}(x) F_{i 62}(x) d x, \\
& \alpha_{n i}^{c}=-\sum_{j=n}^{\infty} \frac{P_{n j}}{P_{j j} I_{j}} \int_{0}^{c} G_{j}(x) F_{i 63}(x) d x, \\
& \alpha_{n i}^{d}=-\sum_{j=n}^{\infty} \frac{P_{n j}}{P_{j j} I_{j}} \int_{0}^{c} G_{j}(x) F_{i 64}(x) d x, \\
& \alpha_{n i}^{f}=-\sum_{j=n}^{\infty} \frac{P_{n j}}{P_{j j} I_{j}} \int_{0}^{c} G_{j}(x) F_{i 66}(x) d x
\end{aligned}
$$


Substituting Equation (A.12) into Equation (A.5), the equality now becomes

$$
\begin{aligned}
& \sum_{n=1}^{\infty} a_{n} F_{n 51}^{*}(x)+\sum_{n=1}^{\infty} b_{n} F_{n 52}^{*}(x)+\sum_{n=1}^{\infty} c_{n} F_{n 53}^{*}(x) \\
& +\sum_{n=1}^{\infty} d_{n} F_{n 54}^{*}(x)+\sum_{n=1}^{\infty} f_{n} F_{n 56}^{*}(x)=-u_{5}^{*}(x) \\
& \text { for }|x| \leq c
\end{aligned}
$$

with

$$
\begin{aligned}
& F_{n 51}^{*}(x)=F_{n 51}(x)+\sum_{i=1}^{\infty} \alpha_{i n}^{a} F_{i 55}(x), \\
& F_{n 52}^{*}(x)=F_{n 52}(x)+\sum_{i=1}^{\infty} \alpha_{i n}^{b} F_{i 55}(x), \\
& F_{n 53}^{*}(x)=F_{n 53}(x)+\sum_{i=1}^{\infty} \alpha_{i n}^{c} F_{i 55}(x), \\
& F_{n 54}^{*}(x)=F_{n 54}(x)+\sum_{i=1}^{\infty} \alpha_{i n}^{d} F_{i 55}(x), \\
& F_{n 56}^{*}(x)=F_{n 56}(x)+\sum_{i=1}^{\infty} \alpha_{i n}^{f} F_{i 55}(x), \\
& u_{5}^{*}(x)=u_{5}(x)+\sum_{i=1}^{\infty} \alpha_{i}^{0} F_{i 55}(x)
\end{aligned}
$$

Using the same procedure, the orthogonal function $H_{n}^{*}(x)$ is constructed from $F_{n 56}^{*}(x)$ as

$$
H_{n}^{*}(x)=\sum_{i=1}^{n}\left(Q_{i n} / Q_{n n}\right) \times F_{i 56}^{*}(x)
$$

where $Q_{i n}$ is the cofactor of the element $g_{i n}$ of $D_{n}$ ", which is defined as

$$
\begin{aligned}
& D_{n} "=\left|\begin{array}{cccc}
g_{11} & g_{12} & \ldots & g_{1 n} \\
g_{21} & & \vdots \\
\vdots & & \vdots \\
g_{n 1} & \cdots \cdots \cdots & g_{n n}
\end{array}\right|, \\
& g_{i n}=\int_{0}^{c} F_{i 56}^{*}(x) F_{n 56}^{*}(x) d x
\end{aligned}
$$

Using Equations (A.14) and (A.16), the coefficient $f_{n}$ can be expressed by $a_{n}, b_{n}, c_{n}$ and $d_{n}$ as follows:

$$
\begin{aligned}
f_{n} & =\beta_{n}^{0}+\sum_{i=1}^{\infty} a_{i} \beta_{n i}^{a}+\sum_{i=1}^{\infty} b_{i} \beta_{n i}^{b} \\
& +\sum_{i=1}^{\infty} c_{i} \beta_{n i}^{c}+\sum_{i=1}^{\infty} d_{i} \beta_{n i}^{d}
\end{aligned}
$$

with

$$
\begin{aligned}
& \beta_{n}^{0}=-\sum_{j=n}^{\infty} \frac{Q_{n j}}{Q_{j j} J_{j}} \int_{0}^{c} H_{j}^{*}(x) u_{5}^{*}(x) d x, \\
& \beta_{n i}^{a}=-\sum_{j=n}^{\infty} \frac{Q_{n j}}{Q_{j j} J_{j}} \int_{0}^{c} H_{j}^{*}(x) F_{i 51}^{*}(x) d x, \\
& \beta_{n i}^{b}=-\sum_{j=n}^{\infty} \frac{Q_{n j}}{Q_{j j} J_{j}} \int_{0}^{c} H_{j}^{*}(x) F_{i 52}^{*}(x) d x, \\
& \beta_{n i}^{c}=-\sum_{j=n}^{\infty} \frac{Q_{n j}}{Q_{j j} J_{j}} \int_{0}^{c} H_{j}^{*}(x) F_{i 53}^{*}(x) d x, \\
& \beta_{n i}^{d}=-\sum_{j=n}^{\infty} \frac{Q_{n j}}{Q_{j j} J_{j}} \int_{0}^{c} H_{j}^{*}(x) F_{i 54}^{*}(x) d x, \\
& J_{n}=\int_{0}^{c}\left[H_{n}^{*}(x)\right]^{2} d x
\end{aligned}
$$

Substituting Equation (A18) into Equation (A12), we obtain the following relation:

$$
\begin{aligned}
e_{n} & =\alpha_{n}^{0^{*}}+\sum_{i=1}^{\infty} a_{i} \alpha_{n i}^{a^{*}}+\sum_{i=1}^{\infty} b_{i} \alpha_{n i}^{b^{*}} \\
& +\sum_{i=1}^{\infty} c_{i} \alpha_{n i}^{c^{*}}+\sum_{i=1}^{\infty} d_{i} \alpha_{n i}^{d^{*}}
\end{aligned}
$$

with

$$
\begin{aligned}
& \alpha_{n}^{0^{*}}=\alpha_{n}^{0}+\sum_{j=1}^{\infty} \beta_{j}^{0} \alpha_{n j}^{f}, \\
& \alpha_{n i}^{a^{*}}=\alpha_{n i}^{a}+\sum_{j=1}^{\infty} \beta_{j i}^{a} \alpha_{n j}^{f}, \\
& \alpha_{n i}^{b^{*}}=\alpha_{n i}^{b}+\sum_{j=1}^{\infty} \beta_{j i}^{b} \alpha_{n j}^{f}, \\
& \alpha_{n i}^{c^{*}}=\alpha_{n i}^{c}+\sum_{j=1}^{\infty} \beta_{j i}^{c} \alpha_{n j}^{f}, \\
& \alpha_{n i}^{d^{*}}=\alpha_{n i}^{d}+\sum_{j=1}^{\infty} \beta_{j i}^{d} \alpha_{n j}^{f}
\end{aligned}
$$

Replacing the coefficients $e_{n}$ and $f_{n}$ in Equations (A.1), (A.2), (A.3) and (A.4) with Equations (A.18) and (A.20), the equality becomes

$$
\begin{aligned}
& \sum_{n=1}^{\infty} a_{n} F_{n 11}^{*}(x)+\sum_{n=1}^{\infty} b_{n} F_{n 12}^{*}(x)+\sum_{n=1}^{\infty} c_{n} F_{n 13}^{*}(x) \\
& +\sum_{n=1}^{\infty} d_{n} F_{n 14}^{*}(x)=-u_{1}^{*}(x) \text { for }|x| \leq b \\
& \sum_{n=1}^{\infty} a_{n} F_{n 21}^{*}(x)+\sum_{n=1}^{\infty} b_{n} F_{n 22}^{*}(x)+\sum_{n=1}^{\infty} c_{n} F_{n 23}^{*}(x) \\
& +\sum_{n=1}^{\infty} d_{n} F_{n 24}^{*}(x)=-u_{2}^{*}(x) \text { for }|x| \leq b
\end{aligned}
$$


$\sum_{n=1}^{\infty} a_{n} F_{n 31}^{*}(x)+\sum_{n=1}^{\infty} b_{n} F_{n 32}^{*}(x)+\sum_{n=1}^{\infty} c_{n} F_{n 33}^{*}(x)$

$+\sum_{n=1}^{\infty} d_{n} F_{n 34}^{*}(x)=-u_{3}^{*}(x)$ for $|x| \leq a$

$\sum_{n=1}^{\infty} a_{n} F_{n 41}^{*}(x)+\sum_{n=1}^{\infty} b_{n} F_{n 42}^{*}(x)+\sum_{n=1}^{\infty} c_{n} F_{n 43}^{*}(x)$

$+\sum_{n=1}^{\infty} d_{n} F_{n 44}^{*}(x)=-u_{4}^{*}(x)$ for $|x| \leq a$

(A.25)

with

$$
\begin{aligned}
& F_{n 11}^{*}(x)=F_{n 11}(x)+\sum_{i=1}^{\infty}\left[\alpha_{i n}^{a^{*}} F_{i 15}(x)+\beta_{i n}^{a} F_{i 16}(x)\right], \\
& F_{n 12}^{*}(x)=F_{n 15}(x)+\sum_{i=1}^{\infty}\left[\alpha_{i n}^{b^{*}} F_{i 15}(x)+\beta_{i n}^{b} F_{i 16}(x)\right], \\
& F_{n 13}^{*}(x)=F_{n 13}(x)+\sum_{i=1}^{\infty}\left[\alpha_{i n}^{c^{*}} F_{i 15}(x)+\beta_{i n}^{c} F_{i 16}(x)\right], \\
& F_{n 14}^{*}(x)=F_{n 14}(x)+\sum_{i=1}^{\infty}\left[\alpha_{i n}^{d^{*}} F_{i 15}(x)+\beta_{i n}^{d} F_{i 16}(x)\right], \\
& u_{1}^{*}(x)=u_{1}(x)+\sum_{i=1}^{\infty}\left[\alpha_{i}^{0^{*}} F_{i 15}(x)+\beta_{i}^{0} F_{i 16}(x)\right] \\
& F_{n 21}^{*}(x)=F_{n 21}(x)+\sum_{i=1}^{\infty}\left[\alpha_{i n}^{a^{*}} F_{i 25}(x)+\beta_{i n}^{a} F_{i 26}(x)\right], \\
& F_{n 22}^{*}(x)=F_{n 25}(x)+\sum_{i=1}^{\infty}\left[\alpha_{i n}^{b^{*}} F_{i 25}(x)+\beta_{i n}^{b} F_{i 26}(x)\right], \\
& F_{n 23}^{*}(x)=F_{n 23}(x)+\sum_{i=1}^{\infty}\left[\alpha_{i n}^{c^{*}} F_{i 25}(x)+\beta_{i n}^{c} F_{i 26}(x)\right], \\
& F_{n 24}^{*}(x)=F_{n 24}(x)+\sum_{i=1}^{\infty}\left[\alpha_{i n}^{d^{*}} F_{i 25}(x)+\beta_{i n}^{d} F_{i 26}(x)\right], \\
& u_{2}^{*}(x)=u_{2}(x)+\sum_{i=1}^{\infty}\left[\alpha_{i}^{0^{*}} F_{i 25}(x)+\beta_{i}^{0} F_{i 26}(x)\right]
\end{aligned}
$$

$$
\begin{aligned}
& F_{n 31}^{*}(x)=F_{n 31}(x)+\sum_{i=1}^{\infty}\left[\alpha_{i n}^{a^{*}} F_{i 35}(x)+\beta_{i n}^{a} F_{i 36}(x)\right], \\
& F_{n 32}^{*}(x)=F_{n 35}(x)+\sum_{i=1}^{\infty}\left[\alpha_{i n}^{b^{*}} F_{i 35}(x)+\beta_{i n}^{b} F_{i 36}(x)\right], \\
& F_{n 33}^{*}(x)=F_{n 33}(x)+\sum_{i=1}^{\infty}\left[\alpha_{i n}^{c^{*}} F_{i 35}(x)+\beta_{i n}^{c} F_{i 36}(x)\right],
\end{aligned}
$$$$
F_{n 34}^{*}(x)=F_{n 34}(x)+\sum_{i=1}^{\infty}\left[\alpha_{i n}^{d^{*}} F_{i 35}(x)+\beta_{i n}^{d} F_{i 36}(x)\right],
$$$$
u_{3}^{*}(x)=u_{3}(x)+\sum_{i=1}^{\infty}\left[\alpha_{i}^{0^{*}} F_{i 35}(x)+\beta_{i}^{0} F_{i 36}(x)\right]
$$$$
F_{n 41}^{*}(x)=F_{n 41}(x)+\sum_{i=1}^{\infty}\left[\alpha_{i n}^{a^{*}} F_{i 45}(x)+\beta_{i n}^{a} F_{i 46}(x)\right],
$$$$
F_{n 42}^{*}(x)=F_{n 45}(x)+\sum_{i=1}^{\infty}\left[\alpha_{i n}^{b^{*}} F_{i 45}(x)+\beta_{i n}^{b} F_{i 46}(x)\right],
$$$$
F_{n 43}^{*}(x)=F_{n 43}(x)+\sum_{i=1}^{\infty}\left[\alpha_{i n}^{c^{*}} F_{i 45}(x)+\beta_{i n}^{c} F_{i 46}(x)\right],
$$$$
F_{n 44}^{*}(x)=F_{n 44}(x)+\sum_{i=1}^{\infty}\left[\alpha_{i n}^{d^{*}} F_{i 45}(x)+\beta_{i n}^{d} F_{i 46}(x)\right],
$$$$
u_{4}^{*}(x)=u_{4}(x)+\sum_{i=1}^{\infty}\left[\alpha_{i}^{0 *} F_{i 45}(x)+\beta_{i}^{0} F_{i 46}(x)\right]
$$

Equations (A.22), (A.23), (A.24) and (A.25) have been already solved for $a_{n}, b_{n}, c_{n}$ and $d_{n}$ in Ref. [17,15]. 